\title{
La fiction à force de réel : Jean-Charles Massera / Édouard Levé
}

\author{
Pascal Mougin \\ Université Sorbonne Nouvelle - Paris 3
}

Édouard Levé et Jean-Charles Massera, deux auteurs français d'une quarantaine d'années ${ }^{1}$, se situent dans cette tendance de la littérature française qui s'illustre par ces « objets littéraires non identifiés » dont les éditions Al Dante, entre autres, se sont fait le laboratoire et dont les éditions P. O. L ou Allia constituent les vitrines. À leur côté, pour ne citer que quelques noms :

1 Jean-Charles Massera est né en 1965 à Mantes la Jolie. Édouard Levé est né en 1965 à Neuilly. Il s'est donné la mort le 15 octobre 2007. 
Olivier Cadiot, Christophe Tarkos, Nathalie Quintane, Christophe Fiat ou encore Patrick Bouvet.

Levé et Massera partagent cette particularité qu'ils se sont fait connaître l'un et l'autre dans le champ artistique avant de publier des textes littéraires. Massera est d'abord un critique d'art, connu pour ses analyses portant sur des artistes contemporains (Pierre Huyghe, Claude Closky, Thomas Hirschhorn). Édouard Levé, lui, est photographe. Dans les deux cas, la continuité est remarquable entre leurs productions dans le domaine artistique (essais critiques de l'un, séries photographiques de l'autre) et leurs propositions littéraires, qui en découlent directement.

\section{Détournements, réappropriations}

Leurs textes peuvent être considérés comme des fictions (au prix peut-être d'une redéfinition de ce mot) et ont à voir, pour rester vague, avec la réalité contemporaine de la manière la plus directe. Les titres de plusieurs de leurs livres en témoignent. De Jean-Charles Massera, mentionnons France, guide de l'utilisateur (P. O. L, 1998), Amour, gloire et CAC 40 (P. O. L., 1999), United Emmerdements of New Order, précédé de United Problems of Coût de la Main-d'OEuvre (P. O. L, 2002), ou encore, tout récemment, $A$ Cauchemar is born (Verticales, 2007). Édouard Levé, dont les séries photographiques s'intitulent successivement Actualités, Reconstitutions, Fictions, est l'auteur de plusieurs textes, dont un Journal (P. O. L, 2004), à entendre non pas au sens de journal intime mais au sens de quotidien de presse. C'est de ce texte qu'il sera principalement question ici. 
Tous deux, à leur manière, réarticulent le rapport entre réel et fiction en littérature d'autant plus facilement que leurs cultures et leurs références se rattachent davantage à l'histoire de l'art et aux pratiques artistiques contemporaines qu'à cette littérature elle-même, qu'ils contribuent un peu à transformer en évitant certaines des apories où se débat une partie de la littérature d'aujourd'hui, spécialement en France, quand elle se veut en prise avec le contexte immédiat et reste toujours plus ou moins prisonnière de deux tentations : inscrire le réel dans la fiction ou ancrer la fiction dans le réel. La démarche de Massera et de Levé consisterait plutôt à transformer le réel en une espèce de fiction de lui-même. Pour cela, il s'agit d'investir des modes de représentation déjà constitués (en l'occurrence un journal, pour Levé), et d'effectuer sur ces représentations existantes un travail de réappropriation réglé. Plutôt, donc, «faire avec» les discours dominants, sans rien y ajouter, les visiter de l'intérieur pour en faire l'expérience objectivante et démystificatrice qui rendra opaque, c'est-à-dire visible, leur transparence habituelle.

C'est là aussi renoncer à la posture d'auteur, à l'utopie d'une voix singulière et inédite au profit d'un simple procédé pratiquement réitérable par chacun, auteur patenté ou simple lecteur.

Très schématiquement, leurs démarches s'opposent en ce que, chez Massera, le travail de réappropriation et de détournement se fait par excès, tandis que chez Levé il se fait par défaut. Massera, sur un événement bien connu ou sur tel fait divers singulier, entremêle le tout-venant des discours et des parlures d'aujourd'hui, qu'ils soient administratifs, législatifs, économiques, journalistiques. Ostensiblement 
copiés-collés, pastichés ou amplifiés, ils sont retournés en une rumeur grotesque, génératrice de fictions cauchemardesques, affolées, et hilarantes ${ }^{2}$.

\section{Évidement, évidence : le Journal d'Édouard Levé}

Édouard Levé procède de manière inverse : par évidement et soustraction plutôt que par surcharge et hybridation. Le Journal est emblématique du procédé. Ce livre d'environ 150 pages est structuré selon les rubriques des grands quotidiens: International, Société, Faits divers, Économie, SciencesTechnologies, Annonces, Météo, Sports, Culture, Guide, Télévision. Chaque rubrique présente un certain nombre d'articles, d'une longueur qui va du simple entrefilet au papier d'une page ou plus. Ces articles procèdent d'une réécriture d'articles réels, empruntés à la presse quotidienne nationale, mais auxquels Levé fait subir un certain nombre de modifications systématiques. Premièrement, effacement de tous les noms propres, remplacés si nécessaire par le terme générique correspondant sur le modèle du titre du livre luimême Uournal : hyperonyme valant pour Libération, Le Monde, etc.); les noms de personnes et les noms de lieux sont remplacés par des périphrases génériques. Deuxième procédé systématique : l'effacement des dates (jour, mois, année) et des repères temporels, avec la mise au présent de toutes les formes verbales ou presque. Enfin, Levé neutralise toute la parlure journalistique et la rhétorique de la mise en relief, de la dramatisation et de l'expressivité : les phrases sont du type

\footnotetext{
2 Voir mon article : "Jean-Charles Massera : une esthétique du "faire avec" », 2005.
} 
sujet-verbe-complément, les énoncés sont constatifs, peu de subordination, quasi absence de connecteurs logiques.

Un procédé simple, tellement simple que le lecteur en vient, au fil de sa lecture, à s'imaginer qu'il est lui-même en train de réécrire un article de journal.

Voici quelques extraits (en capitales, la rubrique de rattachement).

\section{[1] INTERNATIONAL}

a. Une vingtaine de personnes trouvent la mort dans un attentat suicide commis dans un hôtel d'une station balnéaire. Un homme portant un sac à dos rempli d'explosif s'introduit dans le hall d'entrée, et déclenche la charge lorsqu'il se trouve au milieu d'un groupe qui s'apprête à partir en randonnée. La plupart des victimes sont des touristes, mais leur identité et leur nombre exact ne peuvent être déterminés. La violence de la destruction empêche la reconstitution précise des corps. (Journal, p. 7)

b. Suite à une vague d'attentats meurtriers, le gouvernement décide de confier à un groupe électronique de défense le chantier d'un nouveau navire espion. [...] (Journal, p. 12)

c. En vingt ans, le président qui règne sur l'ancienne colonie est devenu le dictateur sanglant d'un pays à la dérive. [...] Uournal, p. 12)

d. Une épidémie de peste pulmonaire fait quatre morts dans le sud du pays. [...] (Journal, p. 17)

\section{[2] SocIÉTÉ}

Un ancien ministre, haut fonctionnaire pendant la dernière guerre durant laquelle il s'illustre par son zèle à organiser la déportation de centaines de victimes d'une même confession religieuse, est condamné à dix ans de réclusion criminelle, ainsi qu'à une amende de plusieurs millions d'unités monétaires. Deux ans plus tard, la justice condamne l'État à payer la moitié de l'amende, estimant celui-ci coresponsable des déportations 
en ayant créé les conditions autorisant un de ses fonctionnaires à faire preuve de zèle. (Journal, p. 47)

\section{[3] FAITS DIVERS}

Un père de famille accompagné de ses trois enfants détourne un bus en menaçant le conducteur à l'aide d'un couteau. Il se fait raccompagner chez lui. La police l'intercepte à sa descente du véhicule. Un expert parle de «décompensation». Uournal, p. 67)

\section{[4] ÉCONOMIE}

Une affaire survenue à l'étranger éclabousse la filiale du premier cabinet d'audit mondial, qui emploie plus de quatrevingt mille salariés dans quatre-vingt-un pays. Ce cabinet a construit son image sur le «capital confiance» et la «présomption de connaissance ». Pour gérer la crise, la maison mère, bientôt imitée par les filiales mondiales, crée une cellule spéciale. [...] (Journal, p. 73)

\section{[5] SCIENCES-TECHNOLOGIES}

Les études récentes sur les origines de l'humanité confirment que les premiers hommes sont partis d'un même continent pour se répartir sur les autres. Plusieurs vagues successives sont observées, mais les spécialistes ne sont pas d'accord sur leur chronologie. (87)

Un satellite constate la plus grande éjection de matière solaire jamais observée sur cet astre. Plusieurs milliards de tonnes sont expulsés à trois millions et demi de kilomètres à l'heure. (Journal, p. 88)

[6] ANNONCES

Loue chambre meublée pour célibataire salarié. Uournal, p. 91)

Sa femme, ses fils et ses petits-enfants, ont la douleur d'annoncer la disparition d'un homme, dont les obsèques se tiendront dans la plus stricte intimité. (Journal, p. 94)

[7] MÉTÉO 
Des perturbations actives circulent dans un flux rapide sudouest. Les pluies et le vent sur le quart Nord-ouest sont soutenus. L'anticyclone centré sur le Sud protège les régions les plus méridionales, où le soleil s'impose. (Journal, p. 103)

\section{[8] SPORTS}

Un homme remporte la victoire finale dans la tournée des quatre tremplins de saut à ski, la plus prestigieuse épreuve de cette discipline comptant pour la coupe du monde, avec un ultime succès dans le dernier concours. (Journal, p. 107)

\section{[9] CULTURE}

L'auteur a quarante-cinq ans. Après une dizaine de romans, dont deux ont connu un véritable succès (prix pour l'un, adaptation cinématographique pour l'autre), il propose un recueil de neuf nouvelles entre nostalgie et humour, désespoir et utopie. (Journal, p. 117)

\section{[10] GUIDE - Cinéma}

La terre est ravagée par des fantômes extra-terrestres. Deux clans aux méthodes opposées les combattent. (Journal, p. 135)

[11] GuIDE - Expositions

Deux cents photographies de cet artiste et écrivain qui a croisé les plus grandes vedettes de son époque, portraiturées avec élégance et nostalgie. (Journal, p. 136)

\section{[12] TÉLÉVISION}

13.00. Journal.

13.45. Météo.

13.50. Série. (Rediffusion.) Un homme est découvert mort dans une cabine téléphonique. La police cherche à savoir qui la victime a contacté. L'inspecteur localise bientôt la petite amie de la victime, une jeune toxicomane, qui nie avoir reçu l'appel. (Journal, p. 144)

Les textes de Levé, on l'a dit, s'inscrivent dans la continuité de sa pratique photographique. Une série en particulier peut être considérée comme la matrice formelle de Journal. Il s'agit des 
Actualités (2001, Galerie Loevenbruck, Paris), un ensemble d'images qui se présentent sous la forme de reconstitutions en studio de photographies de presse.

On reconnaît en partie le modèle démarqué, celui la photo de presse : légendes, cadrage (frontalité, personnages en pieds ou en plan moyen), disposition des groupes et jeux de regards, gestes, postures. Mais d'autres aspects contredisent le modèle et marquent l'écart de la reconstitution: inexpressivité des visages, caractère figé des gestes (aucun dynamisme, aucun effet d'instantané de type basculement, flou de bougé, éclairage au flash), absence de décor, fond gris «neutre», minimalisme des accessoires, uniformité des vêtements, jusqu'à la récurrence des acteurs d'une image à l'autre.

Levé procède ainsi à un travail de neutralisation, de soustraction, de dépouillement ou de généralisation et d'abstraction, qui va de pair avec un travail de dénudation du procédé de la photographie de studio. À défaut d'analyser en détail l'analogie et les différences entre le travail sur l'image et celui sur le texte, disons que le travail sur l'image s'inscrit assez classiquement dans une critique du spectacle, moins au sens d'une critique du langage photo-journalistique que, plus exactement, d'une critique de la codification du réel - sa scénarisation et sa scénographisation - en vue de sa représentation médiatique. Les images de Levé constituent une critique du réel comme prétexte à images.

Le travail sur le texte, quant à lui, fonctionne de manière plus complexe grâce aux procédés mentionnés plus haut : à la fois comme producteur de fiction et déclencheur d'expérience. 


\section{Spectres du monde}

L'effacement des noms propres a pour effet de transformer le monde en une fiction de lui-même. Remarquons d'abord que l'anonymisation produit des effets différents selon le type d'article transformé. Dans certains cas, le référent reste inférable du contexte derrière la périphrase indéfinie ou l'hyperonyme. Voir l'exemple 2: «Un ancien ministre, haut fonctionnaire pendant la dernière guerre»; «victimes [appartenant à] une même confession religieuse»; «plusieurs millions d'unités monétaires »; ou encore : « La corporation des dirigeants d'entreprises » (le Medef). Mais dans d'autres cas, l'énoncé ne peut plus fonctionner, comme dans les avis de décès, dans la mesure où l'effacement $\mathrm{du}$ nom porte précisément sur l'élément rhématique de l'énoncé (l'information qu'il est censé apporter) : «Sa femme, ses fils et ses petits-enfants, ont la douleur d'annoncer la disparition d'un homme » (exemple 6). Ailleurs, l'énoncé d'origine est à peine modifié, comme dans le cas du fait divers (3): "Un père de famille accompagné de ses trois enfants détourne un bus », ou encore les synopsis de fictions télévisées (12) : «Un homme est découvert mort dans une cabine téléphonique. La police cherche à savoir qui la victime a contacté. »

Mais au fil du texte l'anonymisation tend à mettre sur le même plan ces différents types d'énoncés, et très vite Levé parvient à suspendre chez le lecteur l'effet devinette. Loin d'inciter à rétablir le référent problématique, la suspension des désignateurs rigides est exploitée au contraire pour ses vertus propres de déplacement $d u$ regard et de légère défamiliarisation des objets de discours, même dans les cas où ceux-ci restent virtuellement inférables du contexte. Elle 
produit donc un effet d'étrangeté global qui transforme le monde familier du grand quotidien en une fiction spectrale de lui-même, effet d'étrangeté qui force le lecteur à une nouvelle expérience du monde réel, dès lors qu'il cesse de le reconnaître et de le réduire à du bien connu.

Le même effet de réel fantôme est produit dans les cas où Levé n'a pas substitué un groupe indéfini à un désignateur rigide, mais s'est contenté d'effacer des marqueurs de lieux (pays, ville) tout en gardant des descriptions définies au moyen de déictiques (voir les exemples 1b, 1c, 1d). Ici, le référent est présupposé existant, unique et identifiable dans l'univers de savoir censément partagé entre l'énonciateur et le lecteur, sauf que cette présupposition s'opère en force : l'énonciateur «fait comme si » le lecteur disposait des mêmes repères que lui alors que ce n'est pas le cas. D'où, à la lecture, l'impression très ambivalente d'une très grande proximité, puisque le lecteur est immédiatement happé, le temps de l'article, dans un univers de référence supposé connu et désignable déictiquement, et en même temps empreint d'une étrangeté radicale, puisqu'on ne sait pas de quel univers il s'agit.

Une autre conséquence de ces perturbations des modes de désignation et de construction de la référence est de type énonciatif. Le discours produit par la transformation devient susceptible d'être mis au compte de plusieurs énonciations différentes.

Par certains aspects, le texte semble relever d'une énonciation «de terrain»: le maintien de l'article défini en emploi spécifique et un présent qui garde lui aussi par endroit sa valeur déictique et semble désigner des événements contemporains de l'énonciation (d'autant qu'il voisine parfois 
avec des marqueurs temporels pleinement déictiques: "récemment, prochainement»). On croit ainsi lire ce qu'un journaliste écrirait sur les lieux de son reportage à l'intention d'un destinataire aussi proche que lui de la réalité qu'il évoque. Effet de présence donc.

Mais par ailleurs, l'emploi de l'indéfini fait pencher le discours du côté d'une autre forme d'énonciation : comme si nous lisions non pas un article de journal, mais un vague compte rendu du contenu de celui-ci, procédant à une reformulation plus généralisante. Effet d'éloignement donc. Et ce présent de vérité générale, dans un contexte qui est celui de la forme brève, fait par endroit songer à la fable, à l'apologue ou même à l'histoire drôle: "Un nouveau ministre des Affaires étrangères est nommé.» (Journal, p. 18). «Un candidat à l'élection présidentielle poursuit en justice [un journaliste] pour atteinte à la vie privée [...] » (Journal, p. 20).

Mentionnons encore d'autres effets qui contribuent eux aussi à la production d'un réel en apesanteur et comme muet : suspension des orientations argumentatives (où veut en venir l'article?), suspension de la visée pragmatique, et, plus obliquement, certaines absences mystérieuses, telle celle d'une rubrique "politique». S'ajoutent à cela des phénomènes de mise en abyme du texte, à travers la rubrique culture en particulier.

Pour résumer, l'impression produite d'un réel transfiguré en fiction de lui-même tient à deux effets concomitants, bien qu'opposés, de rapprochement et de mise à distance. Le Journal de Levé se présente d'une part comme un compte-rendu des affaires du monde organisé en rubriques familières mais débarrassé des filtres rhétoriques et discursifs qui 
habituellement informent la perception que nous en avons. Il produit ainsi un effet d'immédiateté et de présence. D'autre part, l'impression d'avoir affaire à une sorte " d'idée moyenne » $\mathrm{du}$ quotidien de presse, faite de la superposition de tous les numéros successifs et allégée de la menue monnaie du divers sensible, produit une mise à distance. Mise à distance qu'on pourrait lire comme porteuse de réductions simplistes, de confusion génératrice d'indifférence ou d'incompréhension, mais qui en même temps renforce l'acuité du lecteur vis-à-vis du (journal) réel : instruit par ce journal fictif des invariants génériques du monde vu par la presse, le lecteur sera d'autant plus sensible à la part d'irréductible du monde réel.

Reste que l'entreprise de Levé est bien une manière de produire de la fiction à partir du réel, une fiction «à force de réel », qui à son tour produit une expérience du monde à la faveur de cet écart critique entre le bien connu et ce qu'on en reconnaît dans le texte.

\section{Pour une littérature conceptuelle}

Revenons pour finir sur la notion de procédé. Les modèles de Levé sont certes à chercher du côté de la littérature à contrainte, de Roussel à Perec, deux auteurs auxquels Levé renvoie par ailleurs. Quant à la démarche de réappropriation d'un discours constitué (ici celui du journal), elle est presque constitutive de la littérature: Levé, comme Massera à sa manière, ne font rien d'autre que radicaliser le principe de la polyphonie bakhtinienne, en reprenant après d'autres (on songe à certaines tentatives d'un Denis Roche ou d'un Christian 
Prigent ${ }^{3}$ ) la technique du cut-up et tous les procédés de détournement et de réappropriation théorisés dans les années soixante par Debord et Wolman'.

La singularité de Levé tient toutefois au fait qu'il se revendique prioritairement d'un modèle moins littéraire que plastique, qui est celui de l'art conceptuel américain des années soixante et soixante-dix et l'esthétique relationnelle ${ }^{5}$ des années quatre-vingt-dix. Un modèle qui diffère du modèle littéraire de l'écriture à contrainte principalement en ce que ce dernier ne remet pas fondamentalement en question l'idée même d'auteur, en raison du pari sur les vertus heuristiques de la contrainte : le procédé (le protocole d'écriture fixé a priori) est censé enclencher un processus (le cheminement inventif destiné à satisfaire aux exigences fixées) qui ne serait pas advenu sans lui, et tout ce qui adviendra à la faveur de l'arbitraire de départ sera porté au compte d'une expressivité et d'une singularité d'auteur. Instruits par Raymond Roussel, nous avons pris l'habitude de juger des textes à procédé à l'aune du processus, c'est-à-dire en y cherchant le sursaut d'invention déclenché par la contrainte initiale. Un texte qui ne ferait qu'opérer un programme d'écriture et s'épuiserait totalement dans sa formule générative paraitra pauvre.

Il en va différemment dans le domaine artistique, au moins depuis la «déclaration d'intention» (Declaration of Intent, 1969) de Lawrence Weiner, l'une des figures centrales du conceptualisme américain: « 1. L'artiste peut concevoir

\footnotetext{
3Voir par exemple : Denis Roche, Le Mécrit (Seuil, 1972); Christien Prigent, Power/Powder (Christian Bourgois, 1977), Voilà les Sexes (Luneau-Ascot, 1981), Commencement (P.O.L, 1989).

4 « Mode d'emploi du détournement », 1956.

5 Voir Nicolas BouRriaud, 1999.
} 
l'œuvre. 2. L'œuvre peut être fabriquée. 3. L'œuvre n'a pas besoin d'être faite » (Weiner, cité par Benjamin Buchloh, 1992, p. 199). (Rappelons que l'un des livres de Levé s'appelle précisément OEuvres et consiste dans la description d'environ 250 œuvres «dont l'auteur a eu l'idée, mais qu'il n'a pas réalisées »; 2002, p.7). L'œuvre de l'auteur est ainsi toute entière dans l'idée ou dans sa définition. Son effectuation reste secondaire, non nécessaire, voire suspecte car toujours susceptible, au mieux, d'une fétichisation par le marché et les esthètes, au pire, d'un devenir décoratif pour salon bourgeois. Non que cette effectuation, quand elle a lieu, soit inutile, mais aucune valeur ajoutée n'en est attendue qui serait à mettre au compte d'une créativité, d'une idiosyncrasie ou d'un génie d'auteur. Corollairement, l'œuvre peut être effectuée par quelqu'un d'autre que l'auteur, et en particulier le spectateur (ou, le cas échéant, le lecteur): telle est la leçon du conceptualisme dont s'inspirera l'esthétique relationnelle.

Inscrit dans ce paradigme du ready-made assisté de Marcel Duchamp, le Journal de Levé fait partie de ces textes délibérément dégagés de toute mythologie romantique et de toute mystique de l'écriture. Il ne propose qu'un dispositif simple, un protocole opérable par tous, à peine plus qu'une hypothèse de travail : soit un journal, soit une contrainte de réécriture, observons ce qui se passe, quelle expérience nouvelle se produit, y compris - et surtout - dans les cas où l'effectuation bloque, là où la contrainte du concept initial révèle ses limites, ses contradictions, là où le réel lui résiste. Cette résistance même, autrement dit la frustration ou le démenti d'une anticipation, s'offre comme la condition optimale de l'expérience. 
Le lecteur lit le Journal comme s'il était virtuellement en train de l'écrire. La seule opération de fiction (au sens propre : façonnage, fabrication - c'est le sens du fingere, latin) imputable à l'auteur, est l'invention du procédé qui conditionne toute la suite. Fiction d'un procédé qui, appliqué au réel, permet d'en renouveler l'expérience.

\section{Bibliographie}

Bourriaud, Nicolas. (1998), Esthétique relationnelle, Dijon, Les presses du réel.

Buchloh, Benjamin. (1992), Essais historiques II, Villeurbanne, Art édition.

Debord, Guy-Ernest et Gil J. Wolman. (1956), « Mode d'emploi du détournement », Les Lèvres nues, no 8, mai, p. 2-14.

LEvé, Édouard. (2004), Journal, Paris, P. O. L;

-. (2001), série Actualités, Paris, Galerie Loevenbruck;

- . (2002), OEuvres, Paris, P. O. L.

MASSERA, Jean-Charles. (1998), France, guide de l'utilisateur, Paris, P. O. L;

-. (1999), Amour, gloire et CAC 40, Paris, P. O.;

- (2002), United Emmerdements of New Order, précédé de United Problems of Coût de la Main-d'CEuvre, Paris, P. O. L;

-. (2007), A Cauchemar is born, Paris, Verticales. 
Mougin, Pascal. (2005), « Jean-Charles Massera : une esthétique du "faire avec" », dans Jean BESSIÈRE et Judit MAAR (dir.), Littérature, fiction, témoignage, vérité, actes du colloque de Paris-III de mars-mai 2004, Paris, L'Harmattan, p. 213-222. Prigent, Christian. (1977), Power/Powder, Paris, Christian Bourgois.

Roche, Denis. (1972), Le Mécrit, Paris, Seuil;

—. (1981), Voilà les Sexes, Paris, Luneau-Asco;

—. (1989), Commencement, Paris, P. O. L.

\section{Résumé}

Plutôt qu'inscrire le réel dans la fiction ou ancrer la fiction dans le réel : transformer le réel en fiction en investissant les modes déjà constitués de sa représentation. J. Ch. Massera et É. Levé pratiquent deux formes opposées de détournement et de réappropriation, par excès chez l'un, par défaut chez l'autre. Le Journal de Levé, réécriture d'un quotidien vidé de ses dates et de ses noms propres, ne retient du réel qu'un référent générique et maximaliste, porté par une voix neutre, étrangement vraisemblable: fiction spectrale qui renouvelle l'expérience du réel du fait de cet écart critique entre le trop connu du monde et ce qu'on en reconnaît dans le texte. La démarche, inspirée de l'art conceptuel, tourne le dos à la tradition de la mimésis. 